\begin{tabular}{|c|c|}
\hline Title & Prolonged morphometric study of barnacles grown on soft substrata of hydrogels and elastomers \\
\hline Author(s) & $\begin{array}{l}\text { A hmed, Nafees; Murosaki, T akayuki; Kurokawa, Takayuki; Kakugo, A kira; Y ashima, Shintaro; Nogata, Y asuyuki; } \\
\text { Gong, Jian Ping }\end{array}$ \\
\hline Citation & $\begin{array}{l}\text { Biofouling, 30(3), 271-279 } \\
\text { https://doi.org/10.1080/08927014.2013.863280 }\end{array}$ \\
\hline Issue Date & $2014-03-16$ \\
\hline Doc URL & http:/hdl.handle.net/2115/58122 \\
\hline Rights & $\begin{array}{l}\text { "This is an A ccepted Manuscript of an article published by Taylor \& Francis in Biofouling on 2014, available online: } \\
\text { http://www.tandfonline.com/ 10.1080/08927014.2013.863280." }\end{array}$ \\
\hline Type & article (author version) \\
\hline Additional Information & There are other files related to this item in HUSCA P. Check the above URL. \\
\hline File Information & GBIF-2013-0236.R1_M_G1101-2.pdf \\
\hline
\end{tabular}

Instructions for use 


\section{Prolonged morphometric study of barnacles grown on soft substrates of hydrogels and}

elastomers

Nafees Ahmed ${ }^{\mathrm{a}, \#}$, Takayuki Murosaki ${ }^{\mathrm{b}, \#, \dagger}$, Takayuki Kurokawa ${ }^{\mathrm{b}}$, Akira Kakugo ${ }^{\mathrm{b}}$, Shintaro Yashima $^{a}$, Yasuyuki Nogata ${ }^{c}$, Jian Ping Gong ${ }^{b_{*}}$

${ }^{a}$ Laboratory of Soft \& Wet Matter, Division of Biological Sciences, Graduate School of Science, Hokkaido University, Sapporo,060-0810(Japan);

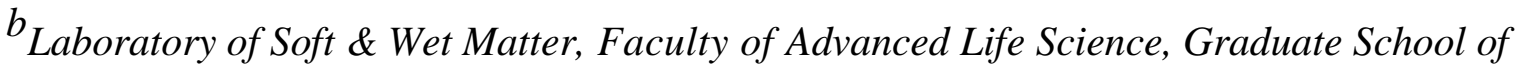
Science, Hokkaido University, Sapporo060-0810(Japan);

${ }^{c}$ Environmental Science Research Laboratory, Central Research Institute of Electric Power Industry, Abiko270-1194(Japan).

†Present address: Bio Device Laboratory, Advanced Institute for Materials Research, Tohoku University, Sendai, 980-8577(Japan);

\#: These two authors make equal contribution.

[*]Corresponding author: Tel/Fax: +81-11-706-2774, E-mail:gong@ mail.sci.hokudai.ac.jp 


\begin{abstract}
A long-term investigation of the shell shape and basal morphology of barnacles grown on tough, double-network (DN) hydrogels and polydimethylsiloxane (PDMS) elastomer were conducted in a laboratory environment. The elastic modulus of these soft substrates varied between 0.01-0.47 MPa. Polystyrene (PS) (elastic modulus, $3 \mathrm{GPa}$ ) was used as a had substrate control. It was found that the shell shape and the basal plate morphology of barnacles were different on the rigid PS substrate compared to the soft substrates of PDMS and DN hydrogels. Barnacles on the PS substrate had a truncated cone shape with a flat basal plate, while on soft PDMS and DN gels, barnacles had a pseudo-cylindrical shape and their basal plates show curvature. In addition, a large adhesive layer was observed under barnacles on PDMS, but not on DN gels. The effect of substrate stiffness is discussed in terms of barnacle muscle contraction, whereby the relative stiffness of the substrate compared to that of the muscle is considered as the key parameter.
\end{abstract}

Keywords: Barnacle, hydrogel, elastic modulus, basal plate, morphology 


\section{Introduction}

Recently, soft substrates are drawing the attention of researchers in the field of marine antifouling materials. An abundance of research related to the behaviour of barnacles on soft substrates, in particular on silicone rubbers, has been conducted. The antifouling (AF) and fouling-release (FR) properties of low elastic modulus polydimethylsiloxane (PDMS) has been revealed and the material has been used for antifouling coatings (Brady et al. 1987; Vincent \& Bausch 1997).

To reveal the FR mechanism of soft materials, basic research on base plate morphology (Berglin \& Gatenholm 2003), the shape of adult barnacles and the characteristics of the adhesives produced (Barlow et al. 2008, Barlow et al. 2010, Kamino 2013) have been reported. Specifically, Berglin and Gatenholm (2003) investigated the evolution of basal plate morphology of barnacles grown on PDMS and poly (methyl methacrylate) (PMMA) during a long-term laboratory assay lasting 3 months. They observed smooth and flat base plates for barnacles grown on PMMA, whereas on a PDMS substrate, they observed featureless base plates with associated thick rubbery material. Wendt et al. (2006) investigated the basal plate morphology of the barnacle Balanus amphitrite in relation to the thickness of silicone coatings immersed in a marine environment for 3 months. No relationship was found between the coating thickness (0.1-2 mm) of PDMS and the frequency of occurrence of the atypical morphology of barnacle basal plates.

Hydrogels, having low elastic modulus and high water content (over $80 \%$ water content by weight) are a class of soft and wet materials. They show high antifouling activities against the settlement of barnacle cypris larvae (Rasmussen et al. 2002, Murosaki et al. 2009a). In previous work, it has been found that some tough hydrogels exhibited high antifouling activities against marine sessile organisms after immersion in marine environment. Furthermore, the adult barnacles grown on the hydrogels were smaller in size and there were differences in the shape of shells and the morphology of the bottom plate compared with those on rigid substrates (Murosaki et al. 2009b). However, this work did not show the evolution of these differences with respect to time.

In a previous paper (Ahmed et al. 2011), a long-term (half year) laboratory assay of barnacles on soft substrates of PDMS and PAMPS/PAAm double network (DN) hydrogels as performed. PAMPS/PAAm DN gels consist of poly(2-acrylamido-2-methylpropanesulfonic acid) (PAMPS) as the first network and polyacrylamide (PAAm) as the second network (Gong et al. 
2003). The elastic modulus of these two kinds of soft substrates was tuned over a wide range of 0.01-0.47MPa. Polystyrene (PS) with an elastic modulus of $3 \mathrm{GPa}$ was used as a rigid control. It was observed that (1) the initial settlement and metamorphosis of cypris larvae dramatically increased with the increase in substrate elastic modulus and no dependence on substrate wettability was found; (2) the growth rate of barnacles on both DN gels and PDMSs did not show a dependence on the elasticity of the soft substrates, but it showed a slightly higher value on the hydrophobic PDMS than on the hydrophilic DN gels; (3) the growth rate on these soft substrates was lower than that on the rigid PS substrate at a late stage of the growth; (4) the "release" of barnacles was observed from PDMS substrate with a modulus higher than 0.01 MPa (Ahmed et al. 2011).

In this paper, the influence of the soft substrates on the shell shape and basal plate morphology of barnacles is reported. Barnacles grown on the three sets of substrates (rigid PS, soft PDMS and DN hydrogels with different elasticity) in the previous long-term laboratory assay were used for the analysis. It was found that the shell shape and base plate of barnacles was substantially influenced by the softness of the substrates within the modulus range investigated.

\section{Materials and methods}

\section{Preparation of the substrates}

Reagents

2-Acrylamide-2-methylpropanesulfonic acid (AMPS) was provided courtesy of Toagosei (Tokyo, Japan), acrylamide (AAm) was purchased from Junsei Chemical Co. Ltd, Tokyo Kasei Kogyo (Tokyo, Japan). $N, N^{\prime}$-methylenebis (acrylamide) (MBAA) (Tokyo Kasei Kogyo, Tokyo, Japan) as a crosslinking agent was purified by recrystallization from ethanol. 2- Oxiglutaric acid (Wako pure Chemicals, Osaka, Japan), an initiator of free radical polymerization, was used as purchased. The precursor of PDMS (Sylgard184 ${ }^{\mathrm{TM}}$ ) resin and the crosslinker of PDMS (catalyst of Sylgard $184^{\mathrm{TM}}$ ) (Dow Corning Toray Co. Ltd.) were used as purchased.

Synthesis of DN gels

PAMPS/PAAm DN gels were synthesized using a two-step sequential network formation technique (Gong et al. 2003). As the first step, PAMPS gel (first network) was synthesized from a $1 \mathrm{M}$ monomer aqueous solution containing $3 \mathrm{~mol} \% \mathrm{MBAA}$ and $0.1 \mathrm{~mol} \%$ 2-oxoglutaricacid by radical polymerization between two glass plates separated by silicone spacer $1 \mathrm{~mm}$ thick. The 
PAMPS gel was immersed in a 2 MAAm aqueous solution containing $0.01 \mathrm{~mol} \% 2$-oxoglutaric acid for at least 1 day until equilibrium was reached. After that, the swollen gel was taken out from the AAm solution and sandwiched by two glass plates and then the second network (PAAm) was subsequently polymerized in the presence of the first network gel (PAMPS) by irradiation with UV light for $8 \mathrm{~h}$. The PAPMS/PAAm DN sample was denoted as PAMPS (1-30.1)/PAAm(2-0-0.01), where the numbers in parenthesis are the concentration of monomer (M), crosslinker ( $\mathrm{mol} \%$ in relative to monomer) and initiator (mol\% in relative to monomer), respectively. A similar method was applied in the case of synthesis PAMPS(1-4-0.1)/PAAm (20-0.01) gel and PAMPS(1-1-0.1)/PAAm(5-0.03-0.01) gel. After gelation, DN gels were immersed in a large volume of autoclaved seawater for approximately 1 week to remove residual chemicals; the autoclaved seawater was changed at least once every day. The resulting thickness of the gels was $\approx 2.0 \mathrm{~mm}$. The PAMPS/PAAm DN gels were colourless and transparent.

\section{Modification of DN gels}

According to the previous study, the modulus of DN gels at small strain decreases with the maximum deformation that the DN gels have experienced, due to the internal fracture of the rigid first network under deformation (Tanaka et al. 2005). According to this result, it is possible to soften the virgin DN gels that have a relatively high modulus to various levels by applying a certain compressive deformation. For this purpose, the parent PAMPS/PAAm DN gels of three different compositions (1-1-0.1/5-0.03-0.01;1-3-0.1/2-0-0.01 and1-4-0.1/2-0-0.01) were cut into disks (15 mm diameter; $\approx 2.0 \pm 0.2 \mathrm{~mm}$ thickness) and were compressed at different strains using a tensile-compressive tester (TensilonRTC-1310A;Orientec, Co.). The strain values applied to obtain the required modulus of the samples is not shown here, but the relationship between the strain values and the degree of softening of DN gels can be found in literature (Nakajima et al. 2013).

After compression, the gels were immersed in autoclaved seawater for 1 day and were then prepared for determination of the elastic modulus. Another set of DN gels was cut into discs with a diameter of $81.5 \mathrm{~mm}$ and were compressed in a similar manner followed by the immersion in autoclaved seawater for one day. The latter DN gels were used for the barnacle settlement and growth investigation.

\section{Synthesis of PDMS elastomer}

PDMS elastomer was prepared according to manufacturer's instructions. After degassing, a mixed solution of the precursor and crosslinker in weight ratios of 1.5, 2.0, 2.5, 3.0, 3.5 and 4.0\% was 
poured in polystyrene (PS) Petri dishes (inner diameter $83.7 \mathrm{~mm}$, height $15 \mathrm{~mm}$ ) (Nunclon ${ }^{\mathrm{TM}}$ delta). The volume was adjusted to keep the thickness about $2 \mathrm{~mm}$. Polymerization was carried out at $60^{\circ} \mathrm{C}$ for $8 \mathrm{~h}$. After polymerization, the PDMS surfaces were washed with autoclaved seawater.

\section{Characterization of substrates}

\section{Elastic modulus of soft substrates}

The elastic moduli of PAMPS/PAAm DN gels and PDMS were determined using a compressive test with a tensile-compressive tester (TensilonRTC-1310A; Orientec Co.). The samples were compressed with two parallel metal plates connected to a load cell at a strain rate of $20 \%$ min. $^{-1}$. The elastic modulus was determined by the average slope of the stress- strain curve over the strain ratio range of $0-0.1$. The elastic modulus measurements would not change the elastic modulus of the DN gels as they were performed at strain values much smaller than the strain values for softening.

\section{Water content of DN gels}

The water content of DN gels was c.a.90 wt\%, as measured by the weight change upon drying using an electronic moisture balance (Shimazu Co., Kyoto, Japan) that evaporates the water in the gel at $150^{\circ} \mathrm{C}$. The detailed procedures are described in Itagaki et al. (2010).

\section{Water contact angle}

The water contact angle on different substrates was measured by applying optical tensiometry (goniometry). The substrate was mounted on the stage of the tensiometer (DropMaster300,

Kyowa Interface Science Co., Saitama, Japan) and a sessile drop of water, $1.0 \mu \mathrm{L}$ in volume, was put on the substrate. The contact angle was measured after15-20 seconds of placing the drop on the substrate. The static water contact angles were $17^{\circ}-22^{\circ}, 60^{\circ}-90^{\circ}$, and $78^{\circ}$ for DN gels, PDMS, and PS, respectively.

In the experiment reported here, all the soft polymers used were prepared on glass substrates. The roughness of the glass was $2-5 \mathrm{~nm}$, and the roughness of hydrogels synthesized on the glass substrates were tens of nanometers, due to the swelling of the gel in water therby increasing the roughness (Kurokawa et al. 2002, Tominaga et al. 2008). Relative to the antennules of cypris larvae of barnacles, which have a roughness of tens of micrometers, the 
hydrogel surfaces can be considered as flat and smooth and thus roughness would not affect settlement and growth of barnacles (Nott 1969).

\section{Preparation of cypris larvae}

The cypris larvae of $B$. amphitrite were cultured according to standard procedures (Yoshimura et al. 2006). Briefly, adult stocks were maintained in plastic $10 \mathrm{~L}$ aquaria with aeration at a controlled temperature of $25^{\circ} \mathrm{C}$, and were fed a daily diet of naupliar larvae of the brine shrimp Artemia salina. Adult barnacles were exposed in the air condition $\left(25^{\circ} \mathrm{C}, 80 \%\right.$ of humidity) for about 12 hours, and then were immersed in the seawater. By this process, nauplii were released from adult barnacles. A concentrated light source facilitated to collection of the photopositive nauplii. Nauplii were collected by pipette and transferred to a beaker at a density of 4 larvae $\mathrm{mL}^{-}$

1 and fed daily with the diatom Chaetoceros gracilis. The concentration of diatoms was kept at $15 \times 10^{4}$ cells $\mathrm{mL}^{-1}$ with a temperature of $25^{\circ} \mathrm{C}$ with a photoperiod of $8 \mathrm{~h}$ light and $16 \mathrm{~h}$ dark. A mixture of streptomycin and penicillin was added to the beaker at the beginning of the culture period to prevent bacterial growth. After 5 days, the culture was filtered through plankton sieves to separate the cypris larvae from the nauplii. Cypris larvae were kept at $6^{\circ} \mathrm{C}$ in the dark in autoclaved seawater for two days before the settlement assays were carried out. Autoclaved seawater was used in all experiments.

\section{Settlement assay}

PS petri dish system

PS Petri dishes (Nunclon ${ }^{\mathrm{TM}}$ Delta) with an inside diameter of $82.5 \mathrm{~mm}$ were used for settlement and growth assays. One set of PS Petri dishes was coated with the parent and modified DN gels (previously cut and compressed) that had been immersed in autoclaved seawater for one day. Another set of PS Petri dishes was coated with PDMS elastomer. As previously shown, agarose gel inhibits settlement of barnacles (Rasmussen et al. 2002, Murosaki et al. 2009a) so it was used as the coating material for the inside wall of PS Petri dished to inhibit settlement on the wall. To apply the agarose coating to the wall of PS Petri dishes, a 4wt\% agarose solution was prepared by dissolving agarose powder in $\mathrm{mQ}$ water and the solution was stirred for $15 \mathrm{~min}$ at $90^{\circ} \mathrm{C}$. The hot agarose solution was first poured in the gap between the inside wall of PS Petri dish and the acrylic mould of $80 \mathrm{~mm}$ in diameter. The dishes were then kept at $4^{\circ} \mathrm{C}$ for $10-$ 15 min to allow the gel to set. After gelation the moulds were removed from the Petri dishes, which were then immersed in a large volume of autoclaved seawater for two days in order to leach out residuals from the agarose gel. The autoclaved seawater was changed twice a day. 
It should be notified that PDMS was directly formed in the PS Petri dishes and it adhered to the bottom of the dishes. On the other hand, DN gels were placed on the bottom of the dishes and they were not bonded to the polystyrene substratum. Thus, the results presented should take account of the different conditions.

\section{Settlement experiment}

After two days immersion in autoclaved seawater, the PS Petri dishes coated with PAMPS/PAAm DN gels and PDMS on the bottom and agarose gel on the walls, were taken out and used for settlement assays with cypris larvae. Bare PS (bottom) was used as controls. As the cypris larvae are photopositive, the outside wall and the lid of dishes were wrapped in black coloured vinyl tape to prevent light penetrating into the Petri dishes. About $7 \mathrm{~mL}$ of autoclaved seawater containing $100 \pm 10$ of 2-day aged cypris larvae were poured into each dish. The dishes were cultured in an incubator (LPH-100S; NK System, Japan) at a temperature $25^{\circ} \mathrm{C}$ with a photoperiod of $8 \mathrm{~h}$ light under a cool white fluorescent lamp and $16 \mathrm{~h}$ dark for 7 days.

Cypris larvae that were shown to explore a suitable surface were used for all the tests. Cypris larvae that had metamorphosed into juvenile barnacles were termed "settled" in this study. A number of cypris larvae were found to settle on the bottom of the dishes coated with DN gels and PDMS, as well as on the bare PS. No cyprids settled on the wall surface covered by the agarose gel.

\section{Observation of the basal morphology and morphometry of barnacles}

Cypris larvae were loaded in PS Petri dishes with various substrates. After 7 days, all cypris larvae that settled on the substrates had metamorphosed into juveniles. The agarose gel coating was then removed from the inside wall of the PS Petri dishes, which were transferred to a $6.5 \mathrm{~L}$ plastic vessel filled with autoclaved seawater. The barnacles were fed with the diatom, Chaetoceros gracilis for one month. The concentration of diatoms in the vessel was maintained at $\approx 25 \times 10^{4}$ cells $\mathrm{mL}^{-1}$ at $25^{\circ} \mathrm{C}$ with a photoperiod of $8 \mathrm{~h}$ light and $16 \mathrm{~h}$ dark. The day of barnacle growth was counted from day 7 after loading the dishes with cyprids. The transparency of the substrates allowed observation of the base plate of barnacles on different substrates; scans were made using a EPSON GT-X700 scanner every two weeks. The size of the barnacles was measured using image-analyzing software, Image-ProPlus6.1. After one month, when the barnacles had a basal diameter of $3 \mathrm{~mm}$, they were fed with nauplii of the brine shrimp, Artemia 
salina. The concentration of the brine shrimp was maintained at about $560 \pm 70$ nauplii $\mathrm{mL}^{-1}$.

As previouslyreported, 10\%-40\% of the barnacles on PDMS substrates spontaneously released from the substrates by day 70 (Ahmed et al. 2011). On the other hand, barnacles did not spontaneously release from the hydrogels. They were pulled off the hydrated gels. Barnacles on PS were peeled off after drying due to strong bonding. The debonded barnacles were used to investigate basal plate morphology. The 3D-profile of dry barnacle basal plates was characterized by using a 3D violet laser-scanning microscope (VK-8700, KEYENCE Co., Ltd.).

\section{Statistical test}

Statistical significance was determined by one way or two way ANOVA followed by Dunnett's test for comparison of substrates with the control. Samples with $p<0.05$ were determined to be statistically significant. In Figure 6b, an unpaired t-test was performed.

\section{Results and discussion}

\section{Shell shape and morphology}

Figure 1 shows typical lateral images of mature barnacles grown on different substrates for 172 days. The barnacles grown on the hard PS substrate have a truncated cone shape with several clear purple lines on the shell wall in the vertical direction. On the other hand, barnacles grown on the soft PDMS and DN gel substrates have a pseudo-cylindrical shape with many indistinct thin purple lines. In comparison to PDMS, barnacles on the DN gels have whitish purple shaded lines.

The shape of barnacles grown on PS, PDMS and DN gels with different elasticity was systematically analysed. Figure 2 shows the relationship between the substrate modulus and shell to substrate angle $(\theta)$ of barnacles on the different substrates. The $\theta$ was found to be $45-65^{\circ}, 80-$ $90^{\circ}$ and $85-95^{\circ}$ for the barnacles on PS, PDMS and DN gels, respectively. Within the elastic modulus range $0.1-0.5 \mathrm{MPa}$ (soft substrates), the value of $\theta$ was almost constant, showing no distinct change with modulus. For the same modulus, $\theta$ is slightly higher on the hydrophilic DN gel substrates than that on the hydrophobic PDMS substrate. However, the $\theta$ on these soft substrates was much higher than that on PS substrate that has a much higher modulus $(E=3 \mathrm{GPa})$. The difference between in the $\theta$ values of barnacles on PS and on the 
soft substrates is statistically significant $(\mathrm{p}<0.01)$. However, the difference between on PDMSs and the DN gels is significant $(\mathrm{p}<0.01$ ) only for substrates with modulus of $0.01,0.09,0.2$, $0.47 \mathrm{MPa}$.

The area of orifice (the area of upper hole of shell wall) and basis of barnacles on the different substrates is shown in Figure 3. The values of orifice area are almost the same (7$\left.10 \mathrm{~mm}^{2}\right)$ on all the substrates, whereas barnacles on PS have a larger area of basis $\left(A_{b}\right)\left(38 \mathrm{~mm}^{2}\right)$ than that on soft substrates $\left(18-22 \mathrm{~mm}^{2}\right)$. The difference in the basal area of barnacles between PS and the soft substrates of PDMS and DN hydrogels is statistically significant $(\mathrm{p}<0.05)$. However, no significant difference of the basal area was observed between samples on PDMS and DN gels.

\section{D morphology of the basal plate}

Previous studies of barnacles grown on hard solid surfaces have revealed two characteristic morphological patterns in the basal plate ie radial lines and concentric rings (Kavanagh et al. 2005, Sangeetha et al. 2010,). Figure 4 shows the typical basal plate morphologies of barnacles grown on PS ( $E=3 \mathrm{GPa}$ ), PDMS and DN (both with $E=0.47 \mathrm{MPa}$ ) at different growth times. Initially, (day 14) there is no clear difference in size and basal morphology between substrates. With the progress of time (day 42), radial lines and concentric rings were observed clearly on the PS substrate, but they were fuzzy (ie indistinct) on the soft substrates. At later times (day 70), the characteristic basal pattern was clearer on PS while it had completely disappeared on the soft substrates. On PDMS especially, a white substance was observed indicating the formation of a white thick adhesive plaque, which was caused by the secretion of adult cement protein as a white gummy mass. The thick adhesive is due to the non-stick feature of the PDMS, as shown in previous reports (Berglin \& Gatenholm 2003, Wiegemann \& Watermann 2003, Sun et al. 2004, Holm et al. 2005), and is in agreement with the easy-release phenomenon of barnacles growing on PDMS (Ahmed et al. 2011). For example, Berglin and Gatenholm (2003) reported that barnacles produce a thick, paste-like white gummy mass on the surface of PDMS. Wiegemann and Watermann (2003) also reported that barnacles on non-stick surfaces typically possessed a bell-shaped base plate and a thick multi-layered adhesive plaque.

To make a quantitative comparison of the $2 \mathrm{D}$ shape of the basal plate, a morphometrical analysis was performed using the parameter of 'form factor' $S$ (Robson et al. 2009). $S$ is defined as $S=4 \pi A_{b} l^{2}$, where $l$ is the perimeter of the basal plate and $A_{b}$ is the basal area. Form factor 
characterises the complexity of the base plate shape: circular shape of the base plate corresponds to a form factor of one, and a deviation from one to a smaller value means that the shape tends to become pseudo-circular, elliptical or even a more irregular shape. Furthermore, the form factor is also influenced by the smoothness of the edge of the base plate. The smoother the edge, the higher the form factor will be. Figure 5 shows the form factor of the basal plate on different substrates. There is no significant difference in the $2 \mathrm{D}$ shape of barnacles on the different substrates. It should be mentioned that the analysis was performed only to barnacles that were settled in isolated state, with no contacting with other barnacles, to avoid any effect from crowded growth (Wethey, 1983).

\section{D morphology of basal plate}

Barnacles grown on PS exhibited a flat morphology; however, those grown on PDMS and DN gel showed a bell-shaped morphology. Figure 6a shows images of the basal plate morphology of barnacles on different substrates. For simplicity, only PDMS and the DN gel of elastic modulus $0.47 \mathrm{MPa}$ are shown. No significant influence of the elasticity of PDMS or DN gel on the architecture of the basis morphology of barnacles was observed.

The curvature of the bell-shaped basal plate was characterized by estimating the ratio of the bell depth, $h$, to the average basal diameter, $d=4 A_{b} / l$. The resulting ratio $h / d$ for the barnacles grown on PS, PDMS and DN $(E=0.47 \mathrm{MPa})$ is shown in Figure $6 \mathrm{~b}$. The basal plate of barnacles on PS exhibits a ratio $h / d$ less than $1 \%$ whereas $h / d$ on PDMS and DN are about $5 \%$ and $13 \%$, respectively. These observations indicate that the stiffness and the deformability of the substrates substantially influence the 3D morphology of the basal plate even for substrates with high moduli (MPa). However, due to the small number of samples $(n=4-6)$, only PS and DN gels show a statistically significant difference $(\mathrm{p}<0.01$; t-test), while no significant difference between PS and PDMS was identified.

\section{Discussion}

Barnacles grown on DN gels secreted a lower amount of adhesive than those grown on PDMS, as seen by the colour of the bottom images in Figure 4. We do not consider this difference is related to whether or not the soft substrates were bonded to the PS Petri dish, which gives different bending resistance of the substrates. If the resistance to free bending of the substrate 
stimulates the barnacles to secret more adhesive, then thick adhesive layers should also be observed on the rigid PS substrate since PS have very high bending resistance. It is considered that the different amount of adhesive layers may come from differences in adhesion strength of the barnacles. The weak adhesion of barnacles to PDMS may stimulate a large adhesive layer, while relatively strong adhesion to the DN gels may not have the same effect. This conclusion is in agreement with the fact that easy-release occurs on PDMS but not on DN gels (Ahmed 2011). Previous studies have shown that the initial settlement of cypris larvae is more effectively inhibited on hydrogels than on PDMS (Murosaki et al. 2009a). However, once barnacles settle on hydrogels, very strong bonding to the gels by inter-digitation of the base plate with the hydrogels occurs (Murosaki et al. 2009b). The latter phenomenon observed in marine environment is consistent with the present long-term result from laboratory a laboratory study.

According to Wiegemann and Watermann (2003), the bell-shaped base plate on a nonstick surface is due to the secretion of a thick multi-layered adhesive plaque. The present study also showed the secretion of adult cement protein as a white gummy mass on the PDMS substrate; the bell-shape on PDMS can also be explained by the same mechanism. However, although the barnacles grown on the DN gels showed only a small secretion of adhesive plaque, they showed a larger curvature of the base plate in comparison with those grown on PDMS. Thus, the large bell-shape of the base plate on hydrogels cannot be explained by the thick adhesive plaque, and it is more likely related to the softness and/or deformation of the hydrogel that was not bonded to the PS petri dish. This conclusion, in turn, also suggests that deformation of soft PDMS may also play a role in the formation of the bell-shaped base plate, in addition to the excess secretion of cement protein.

The effect of substrate softness on the shape of shell wall and base plate can be explained from the contraction of muscles during growth. As shown in Figure 7, which is an extension of a previous barnacle growth model (Wiegemann and Watermann, 2003), the muscle of a barnacle inside the shell is connected to the shell wall and base plate. During the growth of the barnacle, the muscle contracts (Tait \& Emmons 1925), exerting contractile stress on the shell wall and the base plate. The maximum contractile stress is roughly of a similar order to that of the elastic modulus of the muscle $E_{\text {mus }}$. The relationship between the elastic modulus of substrate $\left(E_{\text {sub }}\right)$ and the barnacle muscle $\left(E_{m u s}\right)$ is considered to be a key factor in determining the morphology of barnacles. When the substrate is much rigid than the muscle $\left(E_{s u b}>>E_{m u s}\right)$, the muscle can exerts more and more tensile stress on the basis as a result of the excessive contraction. As the muscles are connected with the inside wall of parietal shell, the excessive contraction of the muscle makes the parietal shell incline 
gradually as the barnacle grows, forming a truncated cone shape. This explains the phenomenon observed for barnacles on PS that is much rigid ( $E=3 \mathrm{GPa}$ ) than the muscle (Figures 1 and 2). The high stiffness of PS explains why the basal morphology of barnacles on PS is almost flat. On a soft substrate that has the modulus comparable to or less than that of muscle $\left(E_{\text {sub }} \lesssim E_{m u s}\right)$, contraction of the muscle causes deformation or bending of the substrate. As a result, the muscle could not exert an increase in tensile stress on the parietal shell in order to make it incline to the truncated cone shape. Although $E_{m u s}$ of the barnacle used in this work (Balanus amphitrite) is unknown, it should be similar to that of the giant barnacle, Balanus nubilus, that was reported around 0.6 MPa (Griffiths et al. 1990). This explains why the pseudo-cylindrical shell shape of the barnacle is formed on the soft substrates of PDMS and DN gels that have lower moduli $(E=0.01-$ $0.47 \mathrm{MPa}$ ) than that of $E_{\text {mus }}$ (Figure 1, Figure 2). Moreover, as the soft DN gels were not bonded to the PS Petri dishes, the gels underwent bending by the barnacles' muscle contraction. On the other hand, the PDMS substrate that was bonded to the PS Petri dishes might undergo stretching deformation under muscle contraction. A free bending deformation occurs more easily than a stretching deformation and explains why the slightly higher $\theta$ on DN gels compared to PDMS is observed (Figure 2). The larger curvature of the bell-shape on DN gels compared to PDMS, as shown in Figure 6, may be also related to the difference in the bonding to the PS Petri dish. The contraction of barnacles' muscle might also alter the modulus of the DN gels, as the latter softens with stress by internal fracture.

\section{Conclusions}

Although the PDMS substrates were bonded to the PS Petri dish while DN gels were not, a similar pseudo-cylindrical shell shape of the barnacles was observed on these soft substrates, in contrast to the truncated cone shape on the rigid substrate. These observations indicate that the elastic modulus of the substrates within the range of sub-MPa influences the shell shape of barnacles. The basal plate of barnacles showed a bell-shape morphology on the soft substrates, different from the flat shape on a rigid substrate. In addition, a large amount of adhesive was observed on PDMS but not on DN gels. Although the adhesive layers of cement protein may also contribute to the bellshape formation in the case of PDMS substrate, the bell-shape is predominantly caused by the deformation and/or bending of the soft substrates. This substrate stiffness effect on the shape of barnacles is related to the relative modulus of the substrate $E_{\text {sub }}$ to that of the muscle of the barnacles $E_{\text {mus }}$. When $E_{\text {sub }}$ is smaller than or comparable to $E_{\text {mus }}$, the substrates experience deformation of 
bending (for free-standing substrate) or stretching (for bonded substrate) by muscle contraction. This deformation creates a large upward force of the muscle, which favours the cylindrical growth of the shell wall and the formation of a bell-shape of the base plate.

\section{Acknowledgments}

This research was financially supported by a Grant-in-Aid for Scientific Research (S) (No. 124225006) from Japan Society for the Promotion of Science (JSPS). 


\section{References}

Ahmed N, Murosaki T, Kakugo A, Kurokawa T, Gong JP, Nogata Y. 2011. Long-term in situ observation of barnacle growth on soft substrates with different elasticity and wettability. Soft Matter. 7(16), 7281-7290.

Barlow DE, Wahl KJ. 2008. Optical spectroscopy of marine bioadhesive interfaces.

Ann Rev of Anal Chem. 5:229-51.

Barlow DE, Dickinson GH, Orihuela B, Kulp JL, Rittschof D, Wahl KJ. 2010. Characterization of the adhesive plaque of the barnacle Balanus amphitrite: amyloid-like nanofibrils are a major component. Langmuir. 26(9):6549-56.

Berglin M, Gatenholm P. 2003. The barnacle adhesive plaque: morphological and chemical differences as a response to substrate properties. Colloids Surf B: Biointerfaces. 28:107-117.

Brady RF Jr, Griffith JR, Love KS, Field DE. 1987. Nontoxic alternatives to antifouling paints. J Coat Technol. 59:113-119.

Gong JP, Katsuyama Y, Kurokawa T, Osada Y. 2003. Double-network hydrogels with extremely high mechanical strength. Adv Mater. 15:1155-1158.

Griffiths PJ, Duchateau JJ, Maeda Y, Ptter JD, Ashley CC. 1990. Mechanical characteristics of skinned and intact muscle fibres form the giant barnacle, Balanus nubilus. Pflügers Arch. 415: 554-565.

Holm ER, Orihuela B, Kavanagh CJ, Rittschof D, 2005. Variation among families for characteristics of the adhesive plaque in the barnacle Balanusamphitrite. Biofouling. 21:121126.

Itagaki H, Kurokawa T, Furukawa H, Nakajima T, Katsumoto Y. Gong JP. 2010. WaterInduced Brittle-Ductile Transition of Double Network Hydrogels. Macromolecules. 43 (22): 9495-9500.

Kamino K. 2013. Mini-review: Barnacle adhesives and adhesion. Biofouling. 29: 735-749.

Kavanagh CJ, Quinn RD, Swain GW. 2005. Observations of Barnacle Detachment from Silicones using High-Speed Video. J of Adhes. 81:843-868. 
Kurokawa T, Gong JP, Osada Y. 2002. Substrate Effect on Topographical, Elastic, and Frictional Properties of Hydrogels. Macromolecules. 35:8161-8166.

Murosaki T, Noguchi T, Kakugo A, Putra A, Kurokawa T, Furokawa H, Osada Y, Gong JP, Nogata Y, Matsumura K, Yoshimura E, Fusetani N. 2009a. Antifouling activity of synthetic polymer gels against cyprids of the barnacle (Balanus amphitrite) in vitro. Biofouling. 25:313320.

Murosaki T, Noguchi T, Hashimot K, Kakugo A, Putra A, Kurokawa T, Saito J, Chen YM, Furokawa H, Gong JP. 2009b. Antifouling properties of tough gels against barnacles in a long-term marine environment experiment. Biofouling. 25:657-666.

Nakajima T, Kurokawa T, Ahmed S, Wu WL, Gong JP. 2013. Characterization of internal fracture process of double network hydrogels under uniaxial elongation. Soft Matter. 9, 1955-1966.

Nott JA. 1969. Settlement of barnacle larvae: surface structure of the antennular attachment disk by scanning electron microscopy. Mar Biol. 2: 248-251.

Rasmussen K, Willemsen PR, Østgaard K. 2002. Barnacle settlement on hydrogels. Biofouling. 18(3):177-191.

Robson MA, Williams D, Wolff K, Thomason JC. 2009. The effect of surface colour on the adhesion strength of Elminius modestus Darwin on a commercial non-biocidal antifouling coating at two locations in the UK. Biofouling. 25(3):215-227.

Sangeetha R, Kumar R, Venkatesan R, Doble M, Vedaprakash L, Kruparatnam, Lakshmi K, Dineshram 2010. Understanding the structure of the adhesive plaque of Amphibalanus reticulates. Mater Sci and Eng C. 30 (1): $112-119$.

Saroyan JR, Lindner E, Dooley CA. 1970. Repair and reattachment in the Balanidae as related to their cementing mechanism. Biol Bull. 139: 333-350.

Sun Y, Guo S, Walker GC, Kavanagh CJ, Swain GW. 2004. Surface elastic modulus of barnacle adhesive and release characteristics from silicone surfaces, Biofouling. 20:279-289.

Tait J, Emmons WF. 1925. Experiments and observations on Crustacea. Part VI. The mechanism of massive movement of the operculum of Balanus nubilus. Proc $\mathrm{R}$ Soc Edinburgh. 45, 42-47.

Tanaka Y., Kuwabara R., Yang H. N., Kurokawa T., Gong J. P. and Osada Y. 2005. 
Determination of Fracture Energy of High Strength Double Network Hydrogels. J Phys Chem B. 109(23):11559-11562.

Tominaga T, Takedomi N, Biederman H, Furukawa H, Osada Y, Gong JP. 2008. Effect of substrate adhesion and hydrophobicity on hydrogel friction. Soft Matter. 4:1033-1040;

Vincent HL, Bausch GG. 1997. Silicone fouling release coatings. Nav Res Rev. 4:39-45.

Yoshimura E, Nogata Y, Sakaguchi I. 2006. Simple methods for mass culture of barnacle larvae. Sessile Org. 2:91-94.

Wendt D.E., Kowalke G. L., Kim J. and Singer I. L. 2006. Factors that influence elastomeric coating performance: the effect of coating thickness on basal plate morphology, growth and critical removal stress of the barnacle Balanus amphitrite. Biofouling. 22 (1): 1-9.

Wethey DS.1983. Intrapopulation variation in growth of sessile organisms: natural populations of the intertidal barnacle Balanus balanoides. Oikos. 40:14-23.

Wiegemann, M, Watermann, B. 2003. Peculiarities of barnacle adhesive cured on non-stick surfaces. J Adhes Sci Technol. 17: 1957-1977. 


\section{Figure captions}

Figure 1. Photographs of the shell morphology of barnacles grown on various substrates for 172 days. The barnacle on PS shows a truncated-cone shape with clear purple lines compared to the pseudo-cylindrical shape on PDMS and the DN gel. On PDMS, the purple lines are unclear while on the DN gel, the lines are almost invisible. The modulus of the PDMS and DN hydrogel was 0.47 MPa.

Figure 2.Shell to substrate angle $(\theta)$ of barnacles grown for 172 days on PS, PDMS and DN gel with different elastic moduli. The vertical bar shows the standard deviation of the mean of 2-22 barnacles settled on each substrate. The statistical significance between the substrates was determined by two way ANOVA followed by Dunnett's test for comparison of the substrates with a control. $\mathrm{p}<0.01$ for PS $v s$. all the PDMS and DN hydrogels except PDMS of $0.09 \mathrm{MPa} ; \mathrm{p}<0.01$ for PDMS vs. DN gels of 0.01, 0.09, 0.2, $0.47 \mathrm{MPa}$.

Figure 3. Project area of orifice $A_{o}$ and basis $A_{b}$, of barnacles as a function of elastic modulus, $E$, of different substrates. The vertical bar shows the standard deviation of the mean of 2-22 settled barnacles on each substrate. Data were collected at day 172. The statistical significance between the substrates was determined by one way ANOVA followed by Dunnett's test for comparison of substrates with a control. No statistically significant difference was identified in (a). $\mathrm{p}<0.01$ for PS vs. DN gels of 0.05, 0.21, 0.33, 0.47 MPa, and PDMS of 0.37 MPa; $\mathrm{p}<0.05$ for PS vs. DN gel of 0.09 MPa and PDMS of 0.05, 0.22 $\mathrm{MPa}$ in (b).

Figure 4. Photographs of the basal plate of barnacles grown on different substrates for various lengths of time. Modulus of PDMS and DN hydrogels was $0.47 \mathrm{MPa}$. The number of days was counted from the time that feeding started at 7 days after cypris larvae were added to the substrates.

Figure 5. Form factor $(S)$ for the base plate of barnacles on various substrates with different 
elastic moduli $€$ after 70 days. The vertical bar shows the standard deviation of the mean of 2-15 barnacles on each substrate. Only isolated barnacles were analysed in order to avoid effects caused by crowding. The statistical significance between the substrates was determined by one way ANOVA followed by Dunnett's test for comparison between substrates and a control. No statistically significant difference was identified between samples.

Figure 6. (a) Photographs showing the morphology of basal plates of barnacles grown on various substrates. (b) Ratio between depth $(h)$ and diameter $(d)$ of basal plates for barnacles grown on various substrates. The vertical bar shows the standard deviation of the mean of 46 samples on each substrate. An unpaired t-test showed no significant difference between PS and PDMS; $p<0.01$ for PS vs. DN gels. Modulus of PDMS and DN gel was $0.47 \mathrm{MPa}$.

Figure 7. Proposed mechanism of the effect of substrate stiffness during barnacle growth. The relationship between the substrate modulus $E_{s u b}$ and the barnacle muscle modulus $E_{m u s}$ determines barnacle morphology. On a hard substrate $\left(E_{s u b}>>E_{m u s}\right)$, no substrate deformation occurs by muscle contraction. This favours the lateral growth to a form truncated-cone shape with a flat basal plate. On a soft substrate $\left(E_{s u b}<E_{m u s}\right)$, the substrate deforms by muscle contraction, which favours the vertical growth to form a pseudo-cylindrical shape with a bell shaped basal plate. In this study, the order of elastic modulus is $E_{P S}>E_{m u s}>E_{P D M S}, E_{D N}$. The adult barnacle illustrated is adapted from Saroyan et al. (1970). 